\title{
DÜBLIN
}

Technological University Dublin

ARROW@TU Dublin

\section{Structure and Polymorphism of Biaxial Bent-Core Smectic Liquid Crystal}

\author{
S. Sreenilayam \\ Technological University Dublin \\ M. Nagaraj \\ Trinity College Dublin, Ireland \\ Yuri Panarin \\ Technological University Dublin, yuri.panarin@tudublin.ie
}

See next page for additional authors

Follow this and additional works at: https://arrow.tudublin.ie/engscheleart2

Part of the Electrical and Computer Engineering Commons

\begin{abstract}
Recommended Citation
Lehmann, A. et al. (2012). Structure and polymorphism of biaxial bent-core smectic liquid crystal. Molecular Crystals and Liquid Crystals , vol. 553, pp. 133-139. DOI:10.1080/15421406.2011.609453
\end{abstract}

This Article is brought to you for free and open access by the School of Electrical and Electronic Engineering at ARROW@TU Dublin. It has been accepted for inclusion in Articles by an authorized administrator of ARROW@TU Dublin. For more information, please contact arrow.admin@tudublin.ie, aisling.coyne@tudublin.ie, gerard.connolly@tudublin.ie.

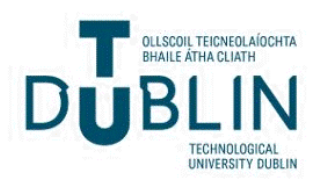




\section{Authors}

S. Sreenilayam, M. Nagaraj, Yuri Panarin, J. K. Vij, A. Lehmann, and C. Tschierske

This article is available at ARROW@TU Dublin: https://arrow.tudublin.ie/engscheleart2/161 


\title{
Structure and Polymorphism of Biaxial Bent-Core Smectic Liquid Crystal
}

\author{
S. SREENILAYAM,${ }^{2}$ M. NAGARAJ,${ }^{1}$ Y. P. PANARIN,${ }^{1,2, *}$ \\ J. K. VIJ, ${ }^{1}$ A. LEHMANN, ${ }^{3}$ AND C. TSCHIERSKE ${ }^{3}$
}

${ }^{1}$ Department of Electronic and Electrical Engineering, Trinity College Dublin, Dublin 2, Ireland

${ }^{2}$ School of Electronic \& Communication Engineering, Dublin Institute

of Technology, Dublin, Ireland

${ }^{3}$ Institute of Chemistry, Organic Chemistry, Martin Luther-University

Halle-Wittenberg, D 06120, Germany

\begin{abstract}
The mesomorphic properties of a homologous series of achiral bent-core compounds are studied by polarizing optical microscopy, electro-optics and polarization measurements. Induction of a new orthogonal smectic phases with the increase of alkyl side chain length was observed. One of the compounds exhibits a unique phase transition between four non-tilted smectic phases $\left(S m A P_{A}-S m A P_{A R}-S m A P_{R}-S m A\right)$. The uniaxial but antiferroelectric nature of $S m A P_{A R}$ phase was confirmed by POM, current response, the 2 nd harmonic electro-optic response and polarization measurements. The structure of SmAP $P_{A R}$ phase was studied theoretically by Next-Nearest-Neighbor model and was identified as $\operatorname{SmAP}_{\alpha}$.
\end{abstract}

Keywords Bent-core molecules; ferroelectricity; biaxial liquid crystal; electro-optic effects; dielectric relaxation

\section{Introduction}

The various sub-phases exhibited by bent-core liquid crystals, their interesting properties and applications, and the existence of polar switching in achiral molecules [1] have attracted the attention of researchers to synthesize and study bent-core compounds in detail. Unlike calamitic liquid crystal compounds, bent-core compounds exhibit a rich variety of orthogonal smectic phases. Some of the recent works in this direction include the study of orthogonal, polar smectic phases $\operatorname{SmAP}_{\mathrm{A}}$ [2-5], $\operatorname{SmA}_{\mathrm{PR}}[6,7]$ and $\mathrm{SmAP}_{\mathrm{AR}}$ [8]. Researchers are also looking for $\mathrm{SmAP}_{\mathrm{F}}$ phase in these materials [9] due to its potential to applications. In all these phases, within a smectic layer, the molecular rotation around the major director is biased resulting in in-layer polarization. Hence, the molecules are packed in the bent-direction which is equivalent to polar axis direction. The common property among these four phases is that they are all non-tilted and possess polar layers.

In the $\mathrm{SmAP}_{\mathrm{R}}$ phase, the in-layer polarizations in smectic layers are randomly arranged, leading to macroscopically uniaxial texture which looks dark between crossed polarizers. In

*Address correspondence to Y. P. Panarin, Department of Electronic and Electrical Engineering, Trinity College Dublin, Dublin 2, Ireland. E-mail: yuri.panarin@ dit.ie 
the $\mathrm{SmAP}_{\mathrm{A}}$ phase, the polarization directions between neighboring layers are anti-parallel leading to a biaxial, antiferroelectric phase.

Under application of strong electric field parallel to smectic layers the initial randomly distributed (in $\mathrm{SmAP}_{\mathrm{R}}$ ) and antiferroelectrically arranged polar directors (in $\mathrm{SmAP}_{\mathrm{A}}$ ) are aligned in the direction of electric field forming macroscopically biaxial ferroelectric structure, $\mathrm{SmAP}_{\mathrm{F}}$. In some materials, the transition from $\mathrm{SmAP}_{\mathrm{A}}$ phase to $\mathrm{SmAP}_{\mathrm{F}}$ phase goes through an intermediate non-birefringent, uniaxial state [10]. Hence these phases are of interest due to their potential application towards next generation displays [11, 12], to understand the internal structure of these phases, molecular structure and property relations [13] etc and thus to realize thermotropic $\mathrm{SmAP}_{\mathrm{F}}$ phase.

Recently, SmAP $\mathrm{PR}_{\mathrm{AR}}$ phase was observed in asymmetrical bent-core compound containing 3-aminophenol central unit [8]. This phase is proposed to consist of local antiferroelectric ordering or domains with long-range randomized polar plane. Asymmetric molecular architectures and the presence of inter molecular hydrogen-bonding are shown to be responsible for stabilizing this phase.

In this manuscript we provide further experimental and theoretical study of the structure of $\mathrm{SmAP}_{\mathrm{AR}}$ phase.

\section{Experimental}

We have studied a homological series of 4-cyanoresorcinol bisbenzoate with two terephthalate-based wings molecules of different terminal chain length by polarizing optical microscopy (POM), electro-optics dielectric and spectroscopy. Homeotropic alignment used in our experiments is obtained by coating AL60702 (JSR Korea) and planar alignment by RN1175 (Nissan Chem., Japan) polymer alignment layers. The electrodes in homeotropic cells were arranged to apply in-plane electric field by etching indium tin oxide (ITO) electrodes on the bottom substrate, distance between the electrodes was $80 \mu \mathrm{m}$.

The structure of studied resorcinol derivatives with different side chain lengths $(n=6$, $8,12,14)$ is shown in Figure 1. Two compounds $(n=6,8)$ exhibit two orthogonal phases: $\mathrm{SmAP}_{\mathrm{A}}-\mathrm{SmA}$. In the next compound $(\mathrm{n}=12)$ new $\operatorname{SmAP}_{\mathrm{R}}$ phase emerges between those two.

The longest material (PAL30, $\mathrm{n}=14$ ) exhibits a new phase sequence (on cooling) $\left(\operatorname{SmAP}_{\mathrm{A}} 95^{\circ} \mathrm{C} \mathrm{SmAP}\right.$ AR $\left.108^{\circ} \mathrm{C} \mathrm{SmAP}_{\mathrm{R}} 117^{\circ} \mathrm{C} \mathrm{SmA} 164^{\circ} \mathrm{C}\right)$ of continuous development of polar order and antiferroelectric property. We could note that the increase of alkyl units in the terminal chains results in formation of an extra smectic phase to the sequence and narrows the $\mathrm{SmAP}_{\mathrm{R}}$ phase range.

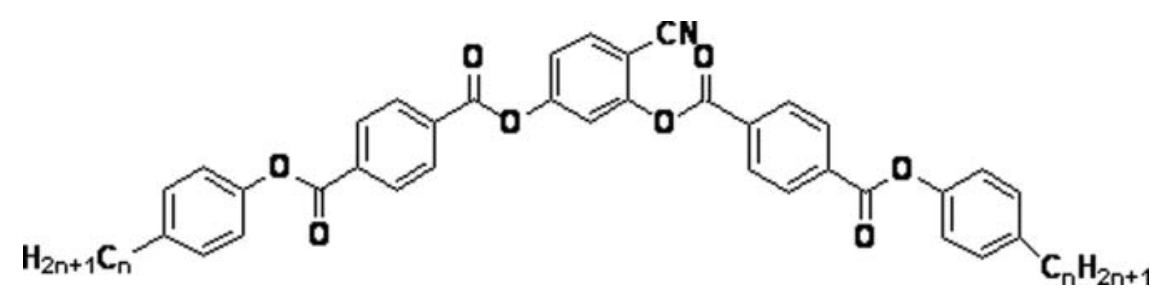

Figure 1. The structure of resorcinol derivatives with different side chain lengths ( $\mathrm{n}=6,8,12,14)$. 


\section{Experimental Study}

\subsection{Biaxiality Study in Homeotropic Cell}

Figure 2 shows the electric field dependence of optical biaxiality (measured in hometropic cell, cell thickness $=7 \mu \mathrm{m}$ ) of PAL30 material in $\mathrm{SmAP}_{\mathrm{R}}, \mathrm{SmAP}_{\mathrm{A}}$ and in $\mathrm{SmAP}_{\mathrm{AR}}$ phases against the electric field applied along the layers.

When homeotropic sample is cooled down from isotropic phase it appears completely dark between the crossed polarizers in both $\mathrm{SmAP}_{\mathrm{R}}$ and $\mathrm{SmAP}_{\mathrm{AR}}$ phases, until it reaches $\mathrm{SmAP}_{\mathrm{A}}$ phase $\left(91^{\circ} \mathrm{C}\right)$, which shows Schlieren texture with both $\frac{1}{2}$ or 1 disclinations. Nevertheless, these two uniaxial phases show different behavior under electric field. In $\mathrm{SmAP}_{\mathrm{R}}$ phase $\left(111^{\circ} \mathrm{C}-108^{\circ} \mathrm{C}\right)$, the applied field disturbs initially randomly distributed polar directors aligning them in the direction of electric field and inducing the biaxial ferroelectric state.

In $\mathrm{SmAP}_{\mathrm{AR}}$ phase $\left(108^{\circ} \mathrm{C}-91^{\circ} \mathrm{C}\right)$ under electric field $\mathrm{E}<1.1 \mathrm{~V} / \mu \mathrm{m}$ it remains dark (uniaxial) and for $\mathrm{E}>1.1 \mathrm{~V} / \mu \mathrm{m}$ the uniaxial state goes to a biaxial state with $\delta \mathrm{n} \approx 0.004$. If the field is further increased to $1.6 \mathrm{~V} / \mu \mathrm{m}$, this biaxial state again goes to a uniaxial state $(\delta \mathrm{n} \approx 0)$, which with the further increase of field $(>1.6 \mathrm{~V} / \mu \mathrm{m})$ again goes to biaxial state $(\delta n \approx 0.015)$ and saturates for higher field. So, this intermediate polar smectic phase under electric field exhibits two macroscopically uniaxial states separated by two biaxial states. The polarization reversal current response of this intermediate phase in a planer cell (thickness $=7 \mu \mathrm{m}$, frequency $=50 \mathrm{~Hz}$ ) showed double peak throughout the temperature range of this phase indicating the antiferroelectric nature of the phase.

In $\operatorname{SmAP}_{\mathrm{A}}$ phase, the initial biaxial anti-ferroelectric structure $(\delta \mathrm{n}=0.017)$ goes to biaxial ferroelectric state $(\delta n=0.025)$ via a non-birefringent uniaxial state $(\delta n=0)$. In PAL30, in $\mathrm{SmAP}_{\mathrm{A}}$ phase these two biaxial states show different values of biaxiality. On

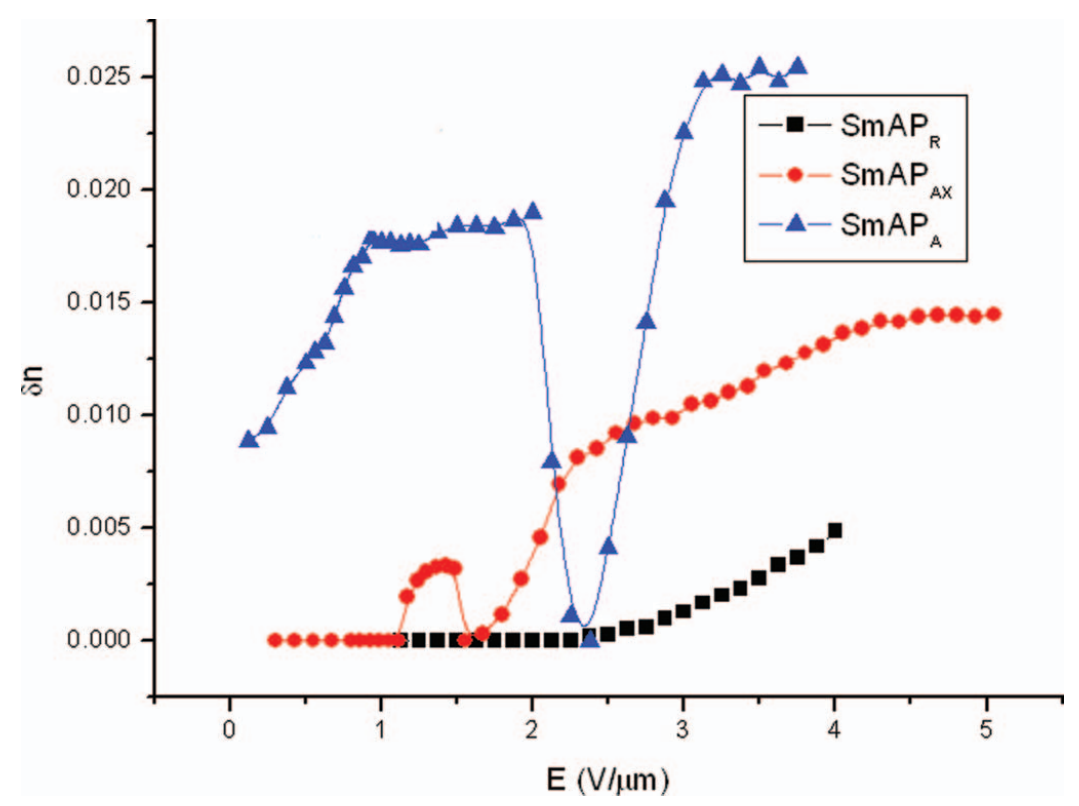

Figure 2. Electric field dependent biaxiality in $\operatorname{SmAP}_{\mathrm{R}}(\boldsymbol{\square}), \operatorname{SmAP}_{\mathrm{AR}}(\bullet), \operatorname{SmAP}_{\mathrm{A}}(\boldsymbol{\Delta})$ phases of PAL30. 
temperature decrease the value of biaxiality in antiferroelectric state increases and may reach the value of field induced ferroelectric state as reported in [10] for another material CK-64 $(\mathrm{n}=12)$. In the intermediate uniaxial state the polar directors are perpendicular to one another in the neighboring layers therefore the average (macroscopic) biaxiality becomes negligible [10]. All the materials $(n=6,8,12,14)$ in their $\operatorname{SmAP}_{A}$ phase show similar type of transformations.

\subsection{Electrooptic Response in Homeotropic Cell}

The electrooptic response was studied in the homeotropic cells described above with help of lock-in amplifier connected to photodiode with the reference from generator. The cells were places between the crossed analyzer and polarizer at $45^{\circ}$ between the applied electric field and the polarizer axes. The samples in all phases show no response on the fundamental harmonic. This is due to the fact that the both positive and negative states are optically equivalent and should show the 2 nd harmonic electro-optic response during the switching. Figure 3 presents the electric field of 2nd-harmonic electro-optic response in $\operatorname{SmAP}_{R}$, $\mathrm{SmAP}_{\mathrm{AR}}$ and $\mathrm{SmAP}_{\mathrm{A}}$ phases.

The magnitude of 2 nd harmonic electro-optic response is proportional to $\sin ^{2}(\pi \cdot \delta n$. $d / \lambda)$. Therefore for $\pi \cdot \delta n \cdot d / \lambda<<1$ and for linear $\delta n(E)$ dependence it would show quadratic dependence on $E$.

The 2nd harmonic electro-optic response $\mathrm{EO}_{2}$ was fitted to equation $E O_{2}=k / E^{\gamma}$ and the exponent $\gamma$ was determined. In both $\operatorname{SmAP}_{\mathrm{R}}$ and $\mathrm{SmAP}_{\mathrm{AR}}$ phases at lower electric fields the exponent $\gamma=2$ which assumes linear $\delta n(E)$ dependence due to the polar interaction

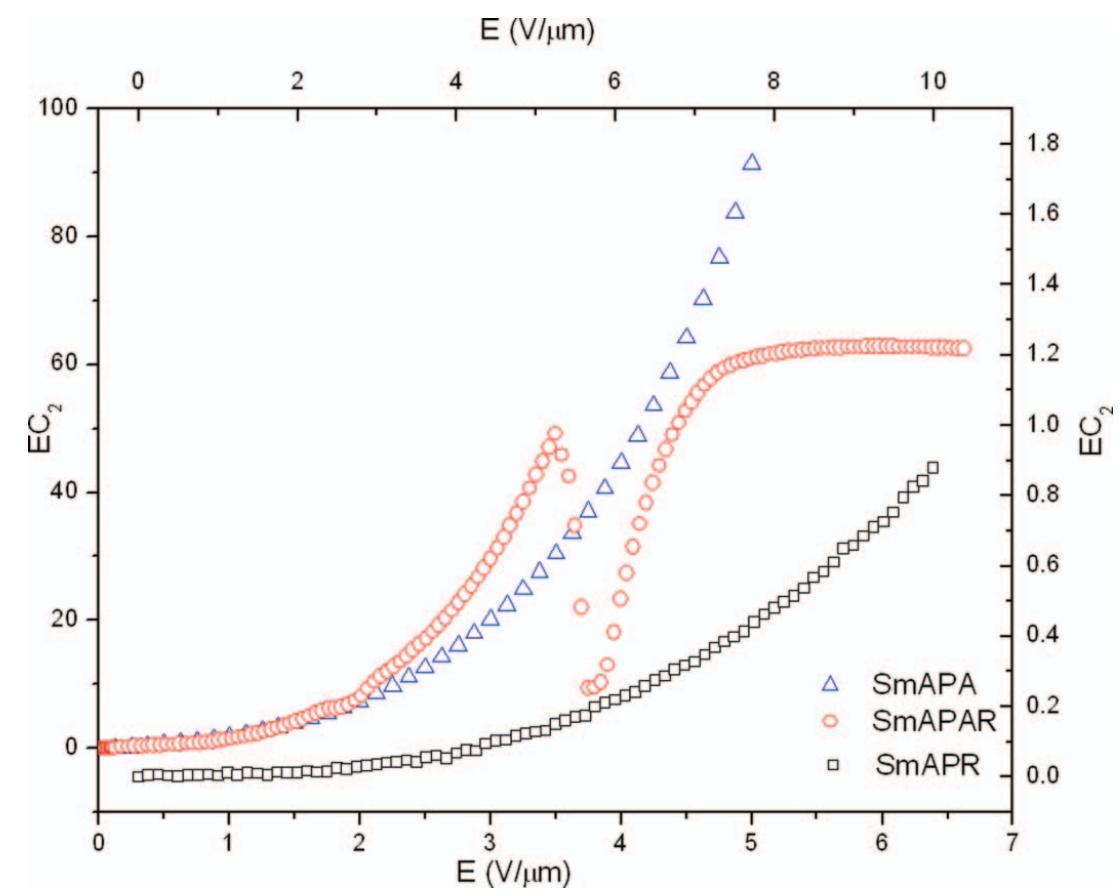

Figure 3. The 2nd harmonic electro-optic response (EC2) in $\operatorname{SmAP}_{R}(\square), \operatorname{SmAP}_{A R}(\boldsymbol{\Lambda})$ and $\operatorname{SmAP}_{A}$ (ム) phases of PAL30. 
with electric field. In $\mathrm{SmAP}_{\mathrm{A}}$ phase the exponent $\gamma$ shows crossover behavior from 2.5 to 3.6, which assumes a combination of polar $(P \cdot E)$ and dielectric $\left(\Delta \varepsilon \cdot E^{2}\right)$ interactions.

\section{Modeling and Discussions}

The component PAL30 $(n=14)$ exhibits four orthogonal smectic phases, three of them with polar smectic layers. The structure of recently discovered $\mathrm{SmAP}_{\mathrm{AR}}$ is still unclear.

To account theoretically for the possible structures we analyze the free energy as a function of polarization in form modified from the nearest neighbor $(\mathrm{NN})$ interactions suggested by Pociecha et al. [14]

$$
G_{N N}=\sum_{j} \frac{1}{2}\left[a_{1 p}\left(p_{j} \cdot p_{j+1}\right)+\frac{1}{4} b_{1 p}\left(p_{j} \cdot p_{j+1}\right)^{2}\right]
$$

The minimization of $\mathrm{G}_{\mathrm{NN}}$ gives rise to five different structures as shown in the Figure 4 [14]:

For $\mathrm{a}_{1 \mathrm{p}}<0$ bilinear interlayer interactions favor ferroelectric order due to Van der Waals attraction between parts of molecules from neighboring layers, whereas for $\mathrm{a}_{1 \mathrm{p}}$ $>0$ antiferroelectric order is stabilized by electrostatic dipolar interlayer interactions. Practically $\mathrm{SmAP}_{\mathrm{F}}$ was rarely observed [9] therefore hereafter we consider $\mathrm{a}_{1 \mathrm{p}}>0$. In this the angle between secondary directors in neighboring layers is obtuse $\left(90^{\circ}<\varphi<180^{\circ}\right)$.

Comparing the possible phase sequence with PAL30 sequence suggests that the $\mathrm{SmAP}_{\mathrm{AR}}$ phase might be either $\mathrm{SmAP}_{\alpha}$ or $\mathrm{SmAP}_{2}$. The $\mathrm{SmAP}_{\alpha}$ is uniaxial and therefore more suitable candidate for $\mathrm{SmAP}_{\mathrm{AR}}$. According to this model both $\mathrm{SmAP}_{\alpha}$ and $\mathrm{SmAP}_{2}$ have the same free energy $\boldsymbol{G}_{N N}$ and therefore are equally possible.

We have modified this model taking into account the next nearest neighbor (NNN) interactions and interaction with electric field, i.e:

$$
G_{N N N}=\sum_{j}\left[\frac{1}{2} a_{1 p}\left(p_{j} \cdot p_{j+1}\right)+\frac{1}{4} b_{1 p}\left(p_{j} \cdot p_{j+1}\right)^{2}+\frac{1}{2} a_{2 p}\left(p_{j} \cdot p_{j+2}\right)-p_{j} \cdot E\right]
$$

In this form, for $\mathrm{a}_{1 \mathrm{p}}>0$ (practical case) at zero electric field the $\mathrm{SmAP}_{\alpha}$ structure is more stable than $\mathrm{SmAP}_{2}$.

The effect of electric was modeled by minimizing $G_{N N N}$ (Eq.2) $\mathrm{a}_{1 \mathrm{p}}=1, \mathrm{~b}_{1 \mathrm{p}}=2, \mathrm{p}_{\mathrm{j}}=$ $1, \mathrm{a}_{2 \mathrm{p}}=\mathrm{a}_{1 \mathrm{p}} / 2, \mathrm{a}_{1 \mathrm{p}} / 4, \mathrm{a}_{1 \mathrm{p}} / 8$ and presented in the Figure 5 . Without electric field the most stable structure is $\mathrm{SmAP}_{\alpha}$ with zero net polarization.

Under application of electric field lower than threshold value $\mathrm{E}_{\mathrm{T}}$, the uniaxial $\operatorname{SmAP}_{\alpha}$ state deforms slightly giving rise to the 2 nd harmonic electrooptic response proportional

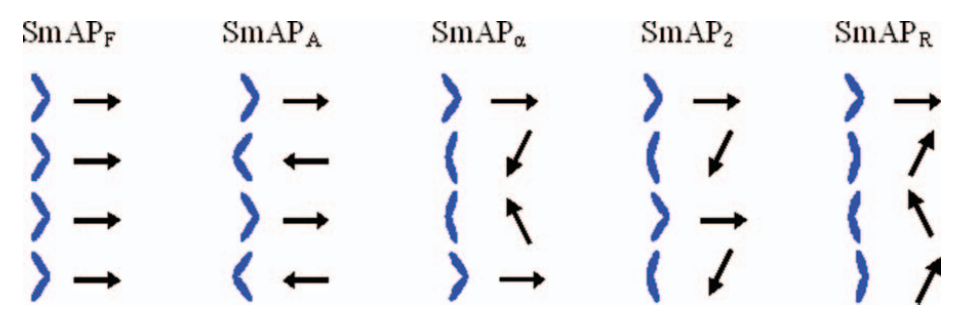

Figure 4. Possible structures in orthogonal smectic phases deduced from the minimization of potential $\mathrm{G}_{\mathrm{NN}}$ (Eq. 1) [15]. 


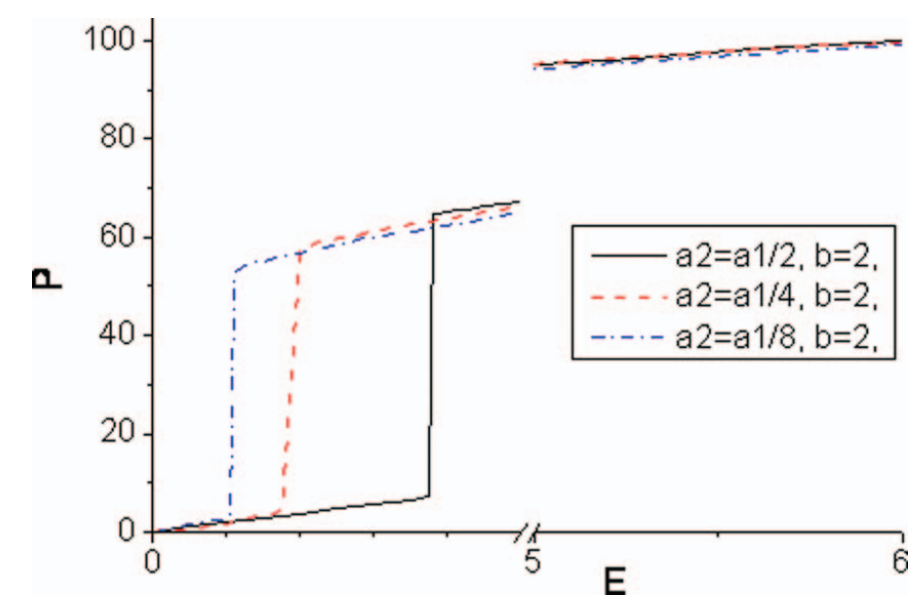

Figure 5. The field-induced normalized polarization. $a_{1 p}=1, b_{1 p}=2, p_{j}=1, a_{2 p}=a_{1 p} / 2, a_{1 p} / 4$, $\mathrm{a}_{1 \mathrm{p}} / 8$.

to the electric field observed experimentally (Fig. 3). Under application of electric field higher than threshold value $\mathrm{E}_{\mathrm{T}}$, the $\mathrm{SmAP}_{\alpha}$ phase becomes unstable and the transition from uniaxial $\mathrm{SmAP}_{\alpha}$ to biaxial $\mathrm{SmAP}_{2}$ phase (with $90^{\circ}<\varphi<180^{\circ}$ ) occurs. This leads to the jump in biaxiality which value nevertheless is smaller than the biaxiality in the field-induced saturated (ferroelectric) state, where $\varphi=0$ and the biaxiality is maximal. On further increase of electric field the angle between secondary directors in neighboring layers gradually changing to zero at saturation field passing though the $90^{\circ}$ which corresponds to uniaxial state. This is in good agreement with the experiment (Fig. 2).

The results of experimental and theoretical studies suggest a possible structure of the $\mathrm{SmAP}_{\mathrm{AR}}$ phase as $\mathrm{SmAP}_{\alpha}$.

\section{Acknowledgment}

The authors thank the EU Grant No. FP7-216025 BIND and SFI Grant No. RFP 06/RFP/ENE039 for funding this work.

\section{References}

[1] Niori, T., Sekine, T., Watanabe, J., Furukawa, T., \& Takezoe, H. (1996). J. Mater. Chem., 6, 1231.

[2] Eremin, A., Diele, S., Pelzl, G., Nadasi, H., Weissflog, W., Salfetnikova, J., \& Kresse, H. (2001). Phys. Rev. E, 64, 051707.

[3] Sadashiva, B. K., Reddy, R. A., Pratibha, R., \& Madhusudana, N. V. (2002). J. Mater. Chem., $12,943$.

[4] Schröder, M. W., Diele, S., Pancenko, N., Weissflog, W., \& Pelzl, G. (2002). J. Mater. Chem., $12,1331$.

[5] Murthy, H. N. S., \& Sadashiva, B. K. (2004). Liq. Cryst., 31, 567.

[6] Pociecha, D., Cepic, M., Gorecka, E., \& Mieczkowski, J. (2003). J. Phys. Rev. Lett., 91, 185501.

[7] Shimbo, Y., Gorecka, E., Pociecha, D., Araoka, F., Goto, M., Takanishi, Y., Ishikawa, K., Meieczkowski, J., Gomola, K., \& Takezoe, H. (2006). Phys. Rev. Lett., 97, 113901.

[8] Gomola, K., Guo, L., Pociecha, D., Araoka, F., Ishikawa, K., \& Takezoe, H. (2010). J. Mater. Chem., 20, 7944. 
[9] Reddy, R. A., Zhu, Ch., Shao, R., Korblova, E., Gong, T., Shen, Yo., Garcia, E., Glaser, M. A., Maclennan, J. E., Walba, D. M., \& Clark, N. A. (2011). Science, 332, 72

[10] Panarin, Y. P., Nagaraj, M., Vij, J. K., Keith, C., \& Tschierske, C. (2010). Europhysics Lett., 92, 26002.

[11] Shimbo, Y., Takanishi, Y., Ishikawa, K., Gorecka, E., Pociecha, D., Mieczkowski, J., Gomola, K., \& Takezoe, H. (2006). Jpn. J. Appl. Phys., 45, L282.

[12] Nagaraj, M., Panarin, Y. P., Vij, J. K., Keith, C., Tschierske, C. (2010). Appl. Phys. Lett., 97, 213505.

[13] Keith, C., Prehm, M., Panarin, Y. P., Vij, J. K., \& Tschierske, C. (2010). Chem. Commun., 46, 3702.

[14] Pociecha, D., Cepic, M., Gorecka, E., \& Mieczkowski, J. (2003). Phys. Rev. Lett., 91, 185501. 